\title{
A Temperature-Based Model for Latent-Period Duration in Stem Rust of Perennial Ryegrass and Tall Fescue
}

\author{
W. F. Pfender
}

USDA-ARS National Forage Seed Production Research Center, Oregon State University Department of Botany and Plant Pathology, 3450

SW Campus Way, Corvallis 97331.

Accepted for publication 17 September 2000.

\begin{abstract}
Pfender, W. F. 2001. A temperature-based model for latent-period duration in stem rust of perennial ryegrass and tall fescue. Phytopathology 91:111-116.

A temperature-response curve for latent-period duration in stem rust (caused by Puccinia graminis subsp. graminicola) on perennial ryegrass and tall fescue was developed from constant-temperature experiments with inoculated plants and evaluated in field experiments. Under constant-temperature conditions, time from infection to $50 \%$ of pustules erupted for perennial ryegrass ranged from 54 days at $3.5^{\circ} \mathrm{C}$ to 5.9 days at $26.5^{\circ} \mathrm{C}$. The latent period $\left(\mathrm{LP}_{50}\right)$ duration of tall fescue was 69 and 8.5 days at these respective temperatures. The dependence of latentperiod completion rate on temperature was best described as a linear

to appear, was used as an estimator of latent-period duration for field observations. Percentage of one latent period completed per half hour (half-hourly rate), for perennial ryegrass was modeled as $(0.0156 T-$ 0.0206) $\{1-\exp [0.497(T-35.5)]\}$, where $T=$ average temperature $\left({ }^{\circ} \mathrm{C}\right)$ during the half-hour period. For tall fescue the modeled rate was $(0.0109 T-0.00214)\{1-\exp [0.417(T-35.5)]\}$. Latent periods modeled by these equations were compared with observed latent periods in field experiments with potted plants, where half-hourly temperatures were measured. Linear regressions of modeled versus observed latent periods had adjusted $r^{2}$ values of 0.96 for perennial ryegrass and 0.93 for tall fescue. The latent-period equations could be used as components of a weather-based disease advisory model to optimize fungicide use in stemrust management on these crops.
\end{abstract} increase in rate with temperature up to approximately $26^{\circ} \mathrm{C}$, then an exponential decline with temperature up to the maximum (lethal) temperature of approximately $35.5^{\circ} \mathrm{C} . \mathrm{LP}_{20}$, the time required for $20 \%$ of open pustules
Additional keywords: Festuca arundinacea, Lolium perenne.
The Willamette Valley of Oregon is the major U.S. production region for certified seed of tall fescue (Festuca arundinacea Schreb.) and all seed of perennial ryegrass (Lolium perenne L.). Stem rust, caused by Puccinia graminis subsp. graminicola, is the greatest disease threat to these grass-seed crops in the region. Stem rust is managed with fungicides, commonly applied at intervals of approximately 21 days. The cost per application of propiconazole fungicide is $\$ 40$ to $\$ 50$ per hectare; perennial ryegrass and tall fescue fields routinely receive two to four and one to three applications, respectively, per season. Because severity of stem rust epidemics varies among years, probably due to weather, the number of pesticide applications used may be greater or fewer than are necessary for efficient disease management. Therefore, a weather-based disease-advisory system would be useful for managing stem rust on grass seed crops.

In modeling the effect of weather on epidemic development, the latent period (LP; time between the start of an infection and sporulation from that infection) is a critical component. For a pathogen with a relatively high multiplication factor latent-period duration is the main determinant of epidemic speed (2) and is considered a component of slow rusting in cereal rust diseases (15). Latent period has been included in simulation models for rust diseases $(11,12)$. Lehman and Shaner (3) observed a latent period variation up to $27 \%$ due to cultivar effect in wheat inoculated with $P$. recondita $\mathrm{f}$. sp. tritici; longer latent periods caused

Corresponding author: W. F. Pfender; E-mail address: pfenderw@ucs.orst.edu

Publication no. P-2000-1019-02R

This article is in the public domain and not copyrightable. It may be freely reprinted with customary crediting of the source. The American Phytopathological Society, 2001. reductions of approximately $85 \%$ in simulated area under disease progress curves. Simulation and field experiments with the same pathogen on Triticale also showed the dramatic effect of latentperiod duration on epidemic development (16).

Within a population of infections there is variation for latentperiod duration, and a graph of cumulative sporulation (number of pustules or spores) over time from a cohort of infections is commonly sigmoid. Such data have been fit to probits $(3,10)$, the Gompertz (13), or the logistic equations (6). Given this variation in latent-period duration among individual infections, an operational definition of the latent-period duration is required. It is often defined as the time required for $50 \%$ of the infections to begin sporulating $\left(\mathrm{LP}_{50}\right)(10)$, but other variations are used (14).

Latent-period duration $\left(\mathrm{LP}_{50}\right)$ is affected by the temperature imposed during latency. The response is generally characterized by a decrease in duration as temperature rises from the minimum to the optimum, then an increasing duration with higher temperatures. Teng et al. (12) described the data for $P$. hordei on barley as $\mathrm{LP}_{50}=$ $19.7-0.085 T^{2}+0.0025 T^{3}$. For alfalfa rust, a linear decrease in logarithm $\left(\mathrm{LP}_{50}\right)$ with temperature from 14 to $30^{\circ} \mathrm{C}$ was observed (13). If, instead of plotting latent-period duration, one plots rate of latent-period completion (i.e., the reciprocal of duration), a temperature-response curve typical of many other life processes is constructed. That is, rate gradually increases with temperature to an optimum, then declines more rapidly to reach zero at the lethal temperature. Several models have been proposed for this type of temperature response curve. Schroedter (9) used a squared sine function of a temperature polynomial to model fungal growth. Analytis (1) developed a more flexible model that can more closely approximate a diversity of temperature response curves. This model produces an equation for relative rate that can conveniently compare different fungi, and requires transforming temperature to a proportion of the range between lower and upper 
limiting temperatures ( 0 to 1 scale) and growth rate to a 0 to $100 \%$ scale (percent of maximum rate). The Analytis equation is: percent of maximum rate $=p t^{\mathrm{n}}(1-t)^{\mathrm{m}}$, where $t=$ transformed temperature, and $p, m$, and $n$ are parameters that are fit to the data. The equation was a good fit to experimental data on mycelial growth rates of several fungi in culture (1). A different model was developed by Logan et al. (5) for temperature response of insectdevelopment rate, with the rationale based on rates of enzymecatalyzed metabolic reactions. They presented a sigmoid exponential function to describe increasing rates up to the temperature optimum, then an exponential decline above the optimum. The model requires fitting three parameters in addition to estimation of the optimum temperature.

Latent-period duration, and its dependence on temperature, has been recorded for $P$. graminis strains on some small grains and grasses. Roelfs (7) noted typical latent periods of 14 to 20 days, and some as long as 30 days, for $P$. graminis f. sp. tritici on wheat in the field. In controlled studies at approximately $20^{\circ} \mathrm{C}$, the same pathogen can have latent periods of 14 days on wheat (6), and 11 to 15 days on various cultivars of barley (4). In a study to design host-resistance tests for stem rust on tall fescue, Welty and Barker (14) observed latent periods of 17.4, 11.8, 10.5, and 10 days at constant temperatures of $10,18,25$, and $27^{\circ} \mathrm{C}$, respectively.

Because there are no data on latent-period duration as a function of temperature for stem rust of perennial ryegrass, and incomplete temperature data for tall fescue, experiments were conducted to determine the relationships. The goal was to produce a model useful for approximating latent-period duration under temperature conditions that can occur in grass seed fields. Such a model may have application in a weather-based disease advisory system for stem-rust management.

\section{MATERIALS AND METHODS}

Two types of experiments were performed to develop a latentperiod duration model and to test it. The temperature-response curves were constructed using data from constant-temperature experiments conducted in growth chambers. The resulting temperature-response model was tested in field experiments with potted plants.

Plants. Perennial ryegrass cv. Morningstar and tall fescue cv. Shenandoah plants for all experiments were grown from seed in a greenhouse supplemented with artificial light (high-pressure sodium lamps, $430 \mathrm{~W}$, Philips Lighting, Somerset, NJ) to provide 14-h day length, and maintained at $21^{\circ} \mathrm{C} / 13^{\circ} \mathrm{C}$ (day/night) until time of inoculation. Plants were grown in vermiculite, watered daily, and fertilized every 2 weeks with $17 \mathrm{mg}$ each of $\mathrm{N}, \mathrm{P}_{2} \mathrm{O}_{5}$ and $\mathrm{K}_{2} \mathrm{O}$ in $25 \mathrm{ml}$ of water. For experiments in growth chambers, 10-week-old plants were used; for field experiments, plants were 10 - to 12 -weeks old when inoculated. Each plant had 10 to 20 tillers when inoculated.

Inoculation procedure. Inoculum, obtained separately for each grass species, was a bulked collection of urediniospores from several different cultivars in a breeder's nursery near Corvallis, OR. Urediniospores were collected with a cyclone sampler and dried overnight at $30 \%$ relative humidity (8), then stored at $-60^{\circ} \mathrm{C}$ for up to 12 months before use. A suspension of $12 \mathrm{mg}$ of spores in $1.0 \mathrm{ml}$ of Soltrol (a light mineral oil) (14), approximately $5 \times$ $10^{6}$ spores per $\mathrm{ml}$, was sprayed onto plants, at $1.0 \mathrm{ml}$ per 50 plants. After allowing the oil to evaporate from leaves (1 to $2 \mathrm{~h}$ ), plants were placed overnight in a dark chamber supplied intermittently with mist $(15 \mathrm{~min} / \mathrm{h})$ that produced a layer of fine water droplets on the leaf surfaces. Temperature in the chamber was held at $18^{\circ} \mathrm{C}$ for $15 \mathrm{~h}$, then fluorescent lights $\left(75 \mu \mathrm{mol}\right.$ photon $\left.\mathrm{m}^{-2} \mathrm{~s}^{-1}\right)$ in the chamber were turned on, mist was applied continuously and the temperature was gradually raised to $25^{\circ} \mathrm{C}$ over the next $4 \mathrm{~h}$ (14). Plants were removed from the mist chamber and placed under the appropriate treatment conditions.
Controlled-temperature experiments. Inoculated plants were placed directly into one of several controlled-temperature growth chambers at one of the following temperatures: $3.5,6.5,12.0$, $20.0,26.5,32.0$, or $34.0^{\circ} \mathrm{C}$. For each trial, there were 10 replicate plants of each species at each temperature tested. There were sufficient growth chambers available to run only three different temperature treatments in each trial, so chambers were reassigned randomly to different temperatures for each trial and a $20^{\circ} \mathrm{C}$ treatment was included in each trial as a standard. Each temperature treatment other than $20^{\circ} \mathrm{C}$ was tested in two to three different trials, and results from all trials for each grass species were used in constructing the temperature-response curve.

Chambers were set for 14.5 -h days at $480 \mu \mathrm{mol}$ photon $\mathrm{m}^{-2} \mathrm{~s}^{-1}$ light at canopy level. Air temperature at canopy level in each chamber was recorded every $10 \mathrm{~min}$ with a thermistor wired to an automated datalogger (CR10X, Campbell Scientific Instruments, Logan, UT). Day and night temperature settings were adjusted to produce measured temperatures that fluctuated by less than $\pm 1.0^{\circ} \mathrm{C}$. Relative humidity in the chambers fluctuated daily from a low of 60 to $75 \%$ to a high of 91 to $97 \%$; average relative humidity over the course of a test varied from 71 to $90 \%$. It was necessary to keep the leaves dry so that additional infections would not occur after the single infection period provided in the mist chamber. To that end, the vermiculite-filled planting containers were placed in a pan of water so that adequate root moisture was maintained without allowing water onto the leaves. There was no dew or guttation fluid observed on leaf surfaces under these growthchamber conditions.

Plants were examined daily with the aid of a $16 \times$ hand lens. The number of open pustules on each plant and the hours elapsed since removing plants from the dew chamber were recorded. A pustule was counted if the plant epidermis over it was cracked open sufficiently for the urediniospores to be observed. Final pustule numbers per tiller varied across trials from 2 to 240 for perennial ryegrass and 1 to 130 for tall fescue.

Analysis and modeling of data from controlled-temperature experiments. For each temperature and trial, the average number of pustules per plant at each assessment time was plotted against elapsed time. For each such data set, the plot of pustule number per time was fit to the logistic or Gompertz equation with the aid of SigmaPlot computer software (SigmaPlot version 4.01, SPSS Inc., Chicago, IL). Appropriateness of each fitted equation was assessed by examining adjusted $r^{2}$ and distribution of standardized residuals. From the fitted equation for each trial, the time between infection (defined as the time when plants were removed from the dew chamber) and eruption of 20 or $50 \%$ of the estimated maximum number of pustules $\left(\mathrm{LP}_{20}\right.$ or $\mathrm{LP}_{50}$, respectively) was calculated.

To construct the temperature-response curve, the rate of development at each temperature was defined as the reciprocal of the time required to reach $\mathrm{LP}_{50}$. Rates from all trials were plotted against the respective growth-chamber temperatures, with temperature calculated as the average of the 10-min readings. Using this plot of rates against temperatures for all trials, an equation was sought to describe the temperature response. The same procedure was used to develop a temperature-response curve for the rate of development to $\mathrm{LP}_{20}$.

Several models were fit to the temperature-response data by least-squares nonlinear regression, and were evaluated based on adjusted $r^{2}$ values and pattern of residuals. Equations were fitted with the use of SigmaPlot software set to 100 iterations and tolerance of 0.000100 . The model of Logan et al. (5), as formulated in their equation 10, is: rate $=a\left\{[1+k \exp (-p T)]^{-1}-\exp (-t)\right\}$, where $T=$ temperature as the independent variable, $t=[(T-$ lethal maximum temperature) / (optimum temperature - lethal maximum temperature)], and $a, k$, and $p$ are parameters. For the model of Analytis (1), I used the following form: relative rate $=p t^{\mathrm{n}}(1-t)^{\mathrm{m}}$, where $t$ is the temperature expressed as a proportion of the range 
between minimum and maximum permissive temperatures for the process. A simpler model was also tested, in which rates for temperatures up to $26^{\circ} \mathrm{C}$ were expressed as a linear function of temperature, and an exponentially decreasing rate above the optimum was described by the factor $1-\exp \left[k\left(T-T_{\max }\right]\right.$, where $T_{\max }$ is the lethal temperature and $k$ is a parameter. This factor is similar to the high-temperature factor in the model of Logan et al. (5).

Field tests of temperature-response model. Growth and inoculation of plants was as described for controlled-temperature experiments. After removal from the mist chamber, plants were taken to the field where the pots were placed in grass plots of the same species. Height of the test plant leaves was adjusted to be even with the top of the field-plant canopy. Field trials were conducted five to six times from March to June in each of 2 years. During each trial average air temperature was measured with a thermistor probe in a standard shield at canopy height (107-L temperature probe, 41301 shield, Campbell Scientific Instruments), and recorded at 30-min intervals for each plot. In any given trial, there was little temperature variation among the four field plots for each grass species, so the average of the four temperature readings was used for each half-hour period. In the 2 years of observations, relative humidity during the field tests ranged from a low of 28 to $40 \%$ to a high of 98 to $100 \%$. Average relative humidity during any one latent period test was between 72 and $88 \%$. Day lengths ranged from 11.5 to $15.5 \mathrm{~h}$ and daily total solar radiation ranged from $8.0 \mathrm{MJm}^{-2}$ in early spring to $51 \mathrm{MJm}^{-2}$ in summer. Maximum daily solar flux ranged between 0.4 and $1.7 \mathrm{Wm}^{-2}$.

For each species, five plants were used in each of four replicate plots (20 plants per species). The plants were examined with the aid of a $16 \times$ lens between 10 a.m. and 2 p.m. every 1 to 2 days, and the first date on which there were at least five erumpent pustules per five-plant replicate was recorded. The beginning of the latent period's conclusion was designated to have occurred 12 or $24 \mathrm{~h}$ previously depending on whether the prior reading had been taken 1 or 2 days before. The observed latent-period duration was calculated as the average from the four plots in each trial. A com- plete record of pustule appearance over time (as was done for the constant-temperature experiments) was not feasible because new infections subsequent to the inoculation procedure would occur in the field and become indistinguishable from the initial infection cohort.

The assumption was made that progress toward completion of the latent period in each half hour can be approximated by the reciprocal of latent-period duration (where latent period is expressed in number of half hours) at the mean temperature during that half hour. With this assumption, I used the temperature-response model to calculate the rate of development (as percentage of the latent period per half hour) for each 30 -min temperature in the field. I then cumulated the percentages for each half-hour until the sum reached $100 \%$. The time elapsed between setting plants in the field and the accumulation of $100 \%$ was the modeled latentperiod duration, which was compared with the latent-period duration derived from direct observation.

\section{RESULTS}

Latent-period duration under constant temperature. For most trials, the data for cumulative pustule appearance over time were fit well by either the Gompertz or the logistic equation. The Gompertz gave a slightly better fit in more cases than the logistic did, as judged by adjusted $r^{2}$ values (all $\geq 0.97$ ) and pattern of standardized residuals. Therefore, all calculations for $\mathrm{LP}_{50}$ and $\mathrm{LP}_{20}$ were based on the Gompertz equation fit to the data. For ease of presentation in Figure 1, the pustule number data for each trial are converted to percentage of maximum pustule number occurring in that trial. The graph for cumulative number of erumpent pustules over time is sigmoid, and steeper at warm and moderate temperatures than it is at cooler temperatures. For both grasses, time from infection to pustule eruption was shortest in the $26.5^{\circ} \mathrm{C}$ treatment, and longer at warmer and cooler temperatures. Pustule eruption was significantly $(P<0.05)$ earlier in perennial ryegrass than in tall fescue at $6.5,12,20,26.5$, and $33^{\circ} \mathrm{C}$. The difference between species was not significant at 3.5 or $32^{\circ} \mathrm{C}$.

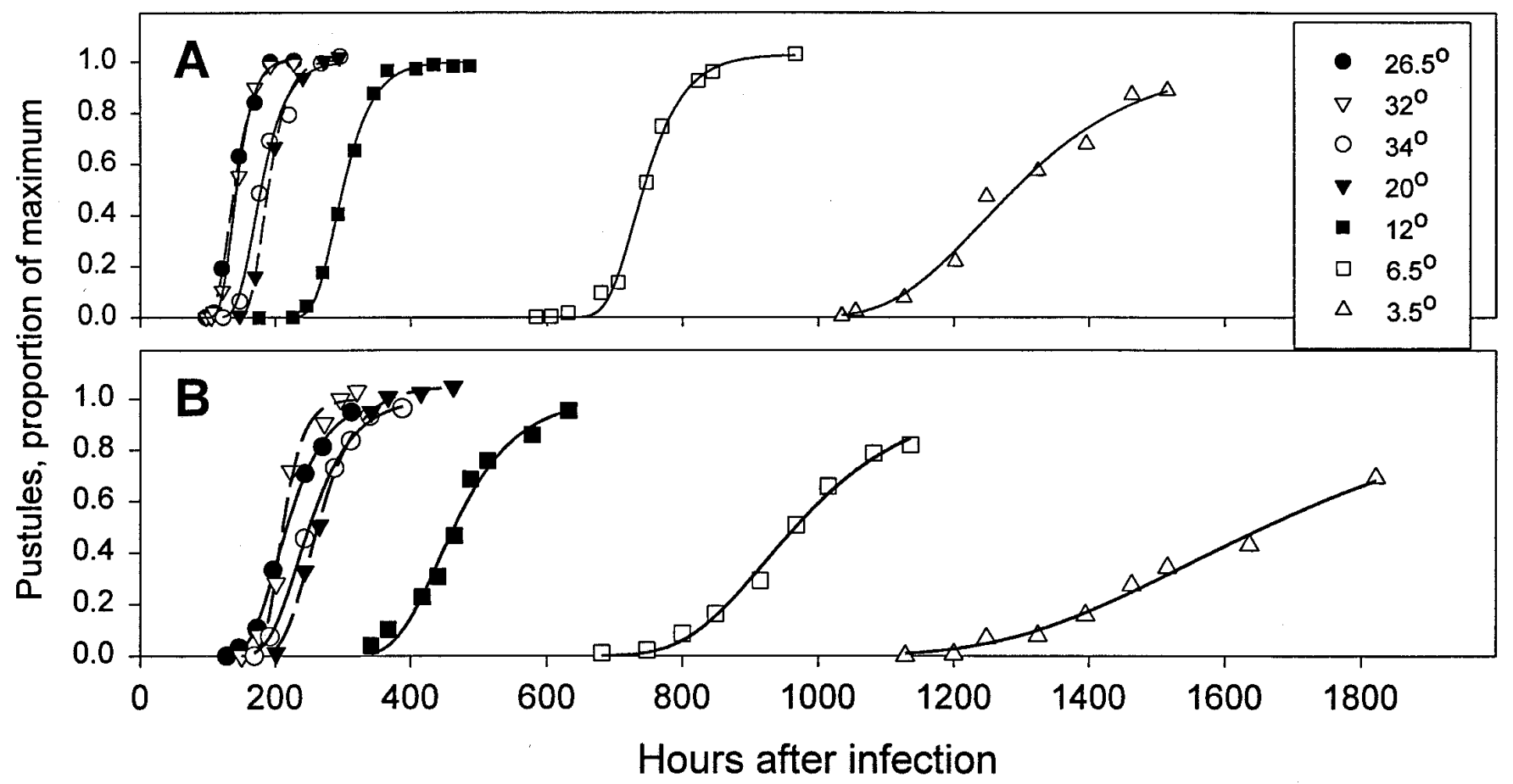

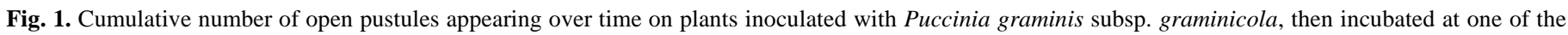

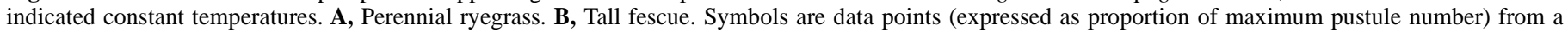
single trial, chosen as an example, at each temperature. Curves are least-squares fit of the Gompertz equation to data points. 
The $\mathrm{LP}_{50}$ at $26.5^{\circ} \mathrm{C}$ is 5.9 days for perennial ryegrass and 8.5 days for tall fescue (Fig. 2). At $3.5^{\circ} \mathrm{C}$, the lowest temperature tested, $\mathrm{LP}_{50}$ is approximately 54 days for perennial ryegrass and 69 days for tall fescue. For each grass species, $\mathrm{LP}_{50}$ values at 32 and $33.5^{\circ} \mathrm{C}$ are longer than they are at $26.5^{\circ} \mathrm{C}$, reflecting typical inhibition of life processes as temperatures approach the lethal maximum. From preliminary experiments at elevated temperatures (data not shown), the lethal maximum temperature for infection development in both grasses was estimated to be between 35 and $36^{\circ} \mathrm{C}$.

Temperature response for developmental rate. Rate for latentperiod completion can be expressed as the reciprocal of latentperiod duration, and the units can be latent period per day, per hour, or other convenient time unit. For the purpose of developing a model to use with automated weather recording stations, a convenient unit for rate is latent period per half hour. This rate can be used directly as the fraction of one latent period completed per half hour at the ambient temperature. The fractions can be summed as temperature records are received, the sum reaching 1.00 at the expected time of latent-period completion. If the $\mathrm{LP}_{50}$ is used for calculating the rate, it is the rate of reaching $50 \%$ of maximum pustule eruption; if the $\mathrm{LP}_{20}$ is used, it is the rate of reaching $20 \%$ of maximum pustule eruption. Although the conventional definition of latent period is $\mathrm{LP}_{50}$ (Fig. $3 \mathrm{~A}$ ), $\mathrm{LP}_{20}$ was also calculated (Fig. 3B) because the goal was to estimate time of occurrence for the first few pustules from an infection event. For each grass species, latent period rates increase approximately linearly with temperature from 3.5 to $26.5^{\circ} \mathrm{C}$. $\mathrm{LP}_{20}$ rates are similar for tall fescue and perennial ryegrass near the temperature minimum, but the perennial ryegrass rate becomes faster than the tall fescue rate as temperatures increase.

Three equations were examined as possible models to fit the $\mathrm{LP}_{20}$ temperature-response data: the model of Logan et al. (5), the model of Analytis (1), and a linear model with an exponential

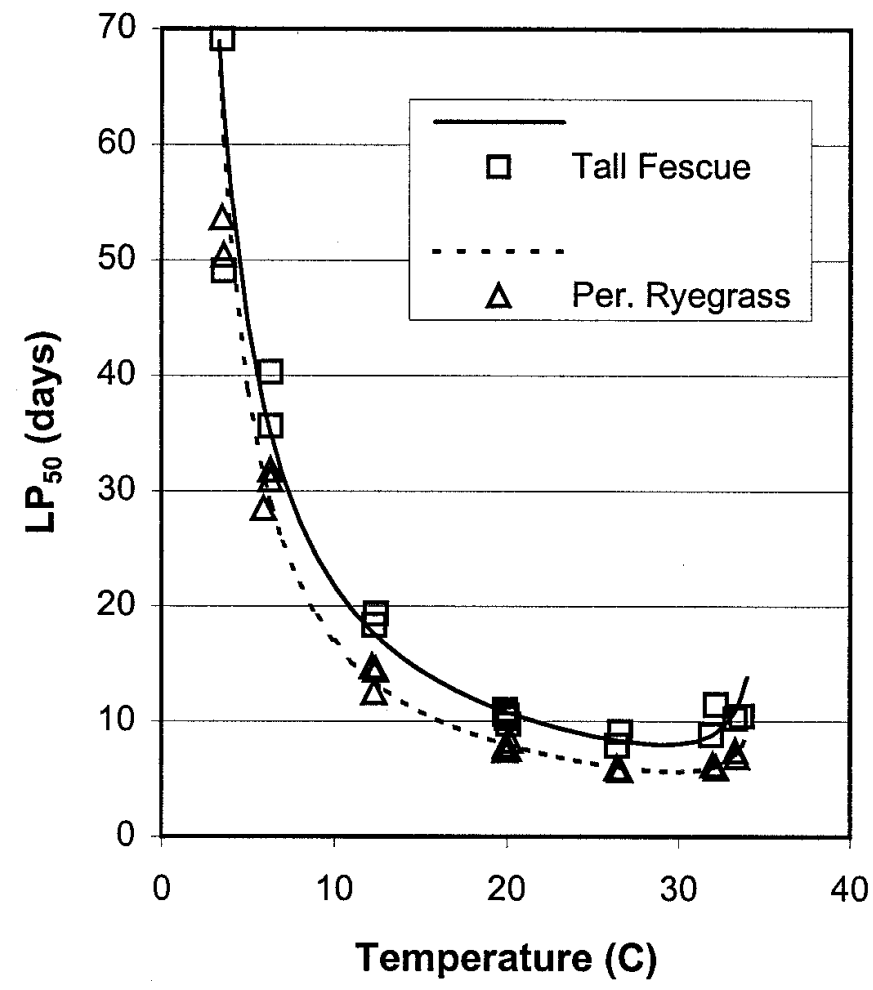

Fig. 2. Latent-period duration expressed as time from infection to $50 \%$ erumpent pustules for stem rust in tall fescue and perennial ryegrass. Data points are $\mathrm{LP}_{50}$ values obtained from fit of the Gompertz equation to individual constant-temperature trials, as in Figure 1. Curves are the reciprocals of leastsquares fit to rate data (Fig. 3). decline above the optimum temperature. For perennial ryegrass all three models were generally acceptable, with adjusted $r^{2}$ values above 0.97 . The form of Logan's equation with fitted parameters was rate $=4.76(1 /\{1+13.9[\exp (-0.16 T)]\}-\exp [-(35.5-T) / 1.8])$. The form of Analytis' equation was relative rate $=10.2 t^{3.97}(1-t)^{0.34}$. Despite the high $r^{2}$ values for these equations, the residuals for the Analytis equation did not appear random, and the Logan equation is very complex, requiring the fitting of four parameters. In contrast, a simpler but quite satisfactory fit $\left(r^{2}=0.99\right)$ was obtained by combining the linear equation for temperatures up to $26.5^{\circ} \mathrm{C}$ with a factor for exponential decline $\left\{1-\exp \left[k\left(T-T_{\max }\right)\right]\right\}$, as the maximum temperature is approached. The form of this equation that best fit the $\mathrm{LP}_{20}$ data was half-hourly rate (as a percent of latent period) $=(0.0156 T-0.0206)$ $\{1-\exp [0.497(T-35.5)]\}$, where $T$ is temperature $\left({ }^{\circ} \mathrm{C}\right)$.

For the tall fescue data, model comparison gave similar results. Standardized residuals from the Analytis equation were patterned, and the better fit of the Logan equation did not justify its complexity. Again the linear equation with exponential decline was selected $\left(r^{2}=0.97\right)$, and its best fit to the tall fescue $\mathrm{LP}_{20}$ data was half-hourly rate $=(0.0109 T-0.00214)\{1-\exp [0.417(T-$ 35.5)]\}.

Latent-period model and field observations. As expected, observed latent periods in the field were longest during cool, early spring weather and shortest in summer. The longest perennial
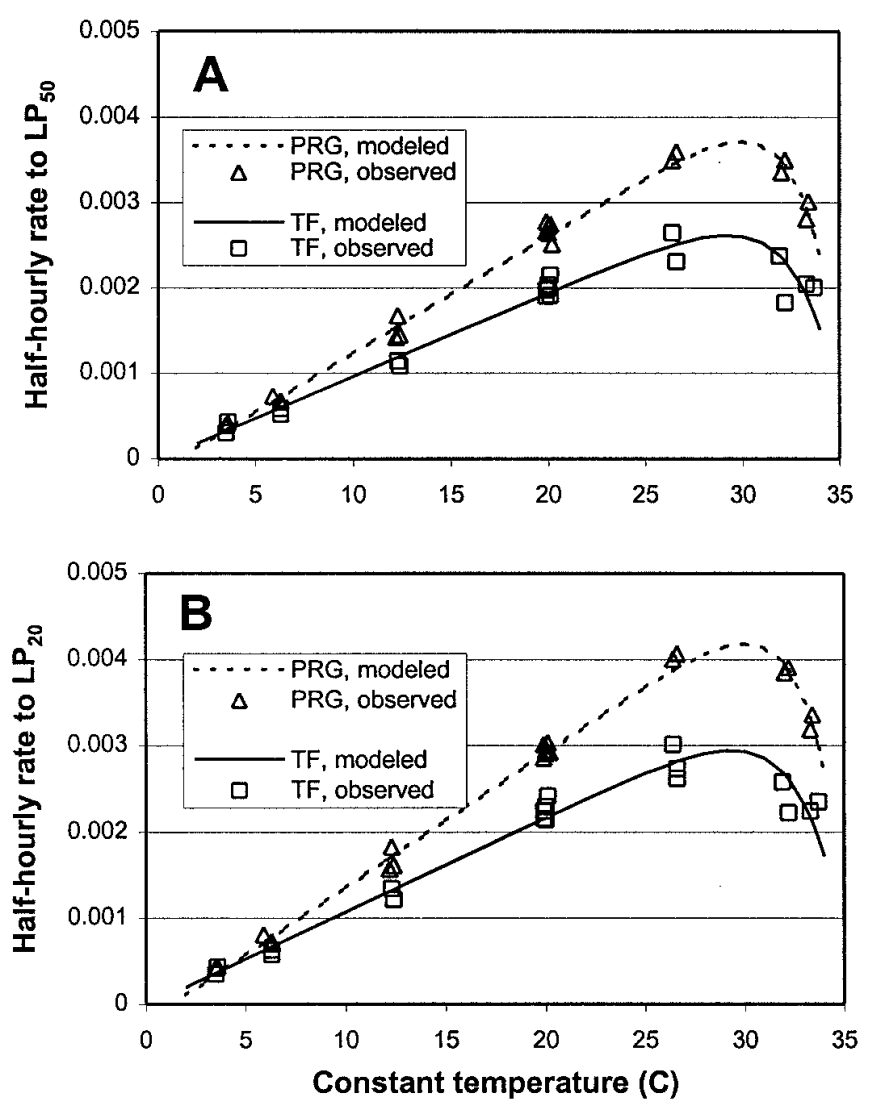

Fig. 3. Latent-period completion rates (percentage of latent period per half hour) as a function of temperature for stem rust in perennial ryegrass and tall fescue. Each data point is calculated from a constant-temperature trial. A, Rate to $50 \%$ of pustules erupted. B, Rate to $20 \%$ of pustules erupted. Curves are least-squares fit for an equation describing a linear increase followed by an exponential decline as temperature approaches the lethal maximum. A, Rate equations for $\mathrm{LP}_{50}$ are: rate for perennial ryegrass (percentage of latent period $/$ half $h)=(0.0138 T-0.0151)\{1-\exp [0.488(T-35.5)]\}$; and rate for tall fescue $=(0.0098 T-0.00209)\{1-\exp [0.406(T-35.5)]\} . \mathbf{B}$, Rate equations for $\mathrm{LP}_{20}$ are rate for perennial ryegrass $=(0.0156 T-0.0206)\{1-$ $\exp [0.497(T-35.5)]\} ;$ rate for tall fescue $=(0.0109 T-0.00214)\{1-$ $\exp [0.417(T-35.5)]\}$, where $T=$ temperature $\left({ }^{\circ} \mathrm{C}\right)$. 
ryegrass latent period observed in the experiments was 24 days (starting 17 March 1999) and the shortest was 8 days (starting 9 June 1999); for tall fescue the range was 26.5 days (17 March 1999) to 11 days (11 June 1998). Temperature values, recorded every $30 \mathrm{~min}$ from sensors near the test plants in the field, were substituted into the equations for perennial ryegrass and tall fescue. These rates, in units of (percentage of one latent period per half hour), were summed starting at the time the plants were placed in the field. The day and time at which the sum reached $100 \%$ was considered the end of modeled latent period. Temperature-response equations fitted to the $\mathrm{LP}_{50}$ data for either grass species consistently overestimated the observed latent periods, whereas equations for the $\mathrm{LP}_{20}$ rates gave good approximations to observed latent period times.

In Figure 4 the modeled and observed latent periods are compared, and the line for modeled $=$ observed is shown. For perennial ryegrass, the model performed well (Fig. 4A). A linear regression analysis of these data (data not shown) produced an adjusted $r^{2}$ of 0.96 and the following relationship: observed latent period $=1.009$ (modeled latent period $)-0.37$. For tall fescue the model performed reasonably well (Fig. 4B), except that the modeled duration for the longest latent period was 4 days shorter than the observed duration for that particular latent period, an underestimate of $13 \%$. The linear regression equation (data not shown) of the tall fescue data was: observed latent period $=1.176$ (modeled latent period) -3.4 , with an adjusted $r^{2}$ of 0.93 .

\section{DISCUSSION}

The observed dynamics of pustule appearance over time are similar to those reported for other rust diseases. The Gompertz or logistic equations, respectively, reportedly gave the best fit for alfalfa rust (13) or wheat stem rust (6) data. For data reported here, the Gompertz was only slightly better than the logistic, and both gave similar results when used to develop estimates of $\mathrm{LP}_{50}$ or $\mathrm{LP}_{20}$

The temperature response of latent-period development rate is most simply described as a linear increase with temperature up to an optimum, followed by exponential decline as the lethal maximum temperature is approached. The model is easy to fit and to apply to temperature data in a datalogger output, where it can be used to give an ongoing estimate of latent-period progress. The Analytis model (1) is more general and may be useful especially for comparison among different fungi, but requires transformation between absolute and relative rates and between absolute and relative temperatures. Also, a pattern in the standardized residuals from regression in my data was apparent for moderate-to-low temperatures. Logan et al.'s model (5) gives a good fit, but is more complex than was needed for the data. Also it requires an estimate of optimum temperature, which cannot be determined accurately without a relatively large number of temperature treatments.

The latent-period durations reported here for tall fescue are similar, but not identical, to those reported by others for a smaller range of temperatures with this species (14). The differences could be due to a higher resolution in measurement and control of growth chamber temperatures in our experiments, or to the use of a different operational definition for latent period (Welty and Barker [14] measured time to $50 \%$ incidence of plants with erupted pustules). The effect of plant cultivar on latent-period duration studied by Welty and Barker (14) may also be involved, although the cultivar differences they noted are not as great as the differences between my estimates and theirs at any given temperature. For eventual implementation of the model described here, host cultivar effects on latent period may be important $(4,14,16)$. This factor is currently under study in related research on tall fescue and perennial ryegrass.

In addition to cultivar effects, there are several factors that might affect the accuracy of this model when applied to a crop in the field. Newton et al. (6) noted that pustule density (pustules/ leaf) can affect dynamics of pustule development, with higher densities producing shorter latent periods than lower densities. An analysis (data not shown) of my data showed no significant correlation of latent-period duration with pustule density over the range of densities I produced in the growth chamber experiments. Variability in latent period due to pathogen genotype (3) also could occur. Nevertheless, I sought to minimize this effect in constructing the model by using a bulk inoculum from many host cultivars, thus including a range of possible pathogen genotypes currently present in the region. Relative humidity or light intensity might influence latent-period duration (and thus model accuracy) under field conditions, although there was no significant correlation of field-test relative humidity with deviation of observed from modeled latent-period durations in my experiments (data not shown). Plant age also could affect latent-period duration, although there is no evidence for this at present.

Another important concern is the precision of a model constructed from experiments with small sample size. As outcrossing species, both perennial ryegrass and tall fescue are genetically heterogeneous and perhaps poorly represented by a 10-plant sample. To investigate sampling effects with respect to latent period, an additional experiment was conducted in which 100 plants of each species were inoculated as described, incubated at $20^{\circ} \mathrm{C}$ and individually scored daily for pustule eruption. Using a random number generator, a 10-plant sample was drawn from the 100plant data set for determination of $\mathrm{LP}_{20}$. This sampling was
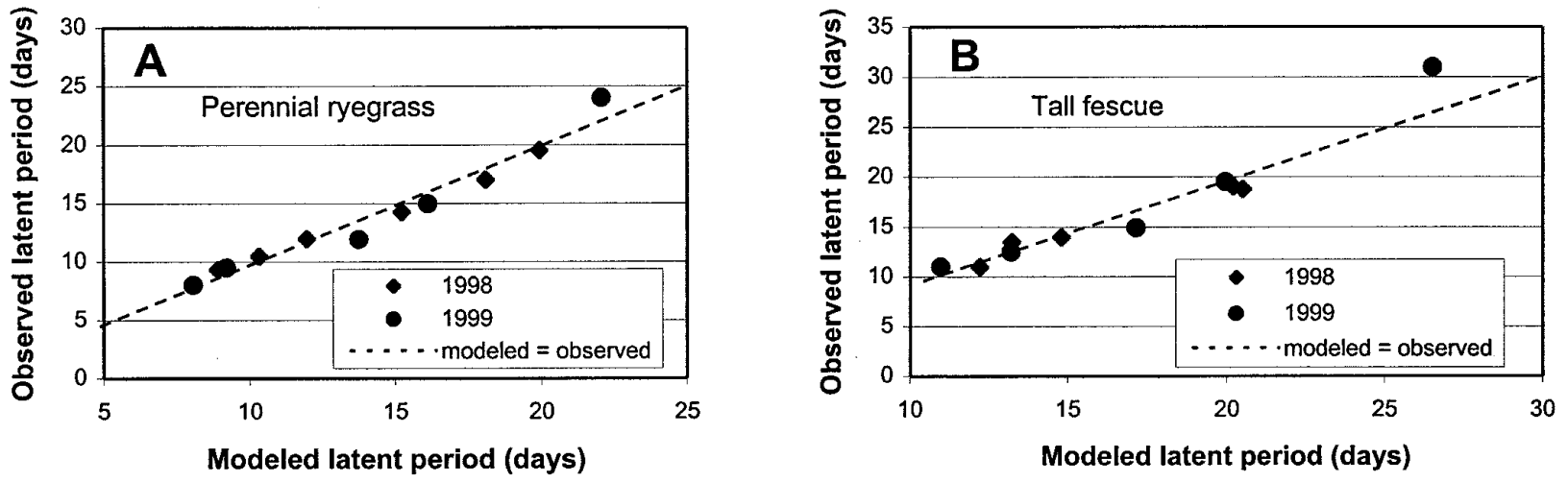

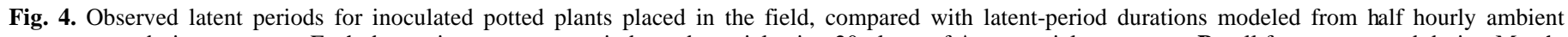

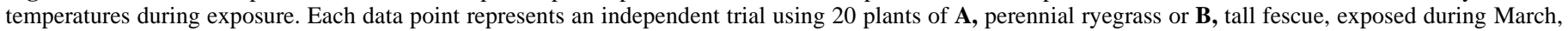
April, May, or June 1998 or 1999. Dashed line indicates position for exact correspondence of observed and modeled values. 
repeated 20 times, replacing values in the data set after each 10 -plant sample. Standard error of the 20 estimates was calculated, and the $\mathrm{LP}_{20}$ estimates were compared with the $\mathrm{LP}_{20}$ calculated from the full 100-plant data set (assumed to provide a good estimate of the true $\mathrm{LP}_{20}$ ). For perennial ryegrass, the 10-plant samples produced $\mathrm{LP}_{20}$ values that ranged from 96 to $105 \%$ of the 100 -plant value, with a standard error of 3.98 h. For comparison, standard error calculated from the several $\mathrm{LP}_{20}$ estimates obtained in repeated $20^{\circ} \mathrm{C}$ standard treatments for the constanttemperature experiments was $3.84 \mathrm{~h}$. For tall fescue the 10-plant samples produced $\mathrm{LP}_{20}$ values that were 91 to $113 \%$ of the 100 -plant calculation, with a standard error of $10.3 \mathrm{~h}$ (compare with $11.5 \mathrm{~h}$ standard error for the $\mathrm{LP}_{20}$ values from the repeated $20^{\circ} \mathrm{C}$ treatments). These data for both species indicate that 10-plant samples for $\mathrm{LP}_{20}$ provide results similar to those from a 100-plant sample. Therefore, a 10-plant sample appears adequate for this research.

Despite the described potential sources of error, the simple temperature-based model performed well (Fig. 4). The least accurate model estimates for observed latent periods appear to be those at the lowest temperatures, i.e., the latent periods of longest duration.

Because the temperature response relationship is essentially linear, at least up to approximately $28^{\circ} \mathrm{C}$, latent period progress can be estimated also by means of a degree-day calculation from daily maximum and minimum temperatures. A double-sine calculation with lower and upper thresholds of 1 and $28^{\circ} \mathrm{C}$, respectively, models a complete latent period in approximately 146 degree days for perennial ryegrass and 180 for tall fescue (data not shown). When temperatures are above $28^{\circ} \mathrm{C}$, however, this degree-day model will not perform well. In the Willamette Valley summer temperatures are rarely above $28^{\circ} \mathrm{C}$ for significant time periods, so a degree-day estimate would be accurate for most of the typical growing season there.

\section{ACKNOWLEDGMENTS}

I thank S. Seguin for excellent technical assistance in this research.

\section{LITERATURE CITED}

1. Analytis, S. 1977. Uber die relation biologischer entwicklung und temperatur bei phytopathogenen pilzen. Phytopathol. Z. 90:64-76.

2. Campbell, C. L., and Madden, L. V. 1990. Introduction to Plant Disease Epidemiology. John Wiley \& Sons, New York.

3. Lehman, J. S., and Shaner, G. 1996. Genetic variation in latent period among isolates of Puccinia recondita f. sp. tritici on partially resistant wheat cultivars. Phytopathology 86:633-641.

4. Liu, J. Q., and Harder, D. E. 1996. Components of resistance in barley to stem rust: Receptivity, urediniospore production, latent period, and infection response in adult plants. Can. J. Bot. 74:1298-1304.

5. Logan, J. A., Wollkind, D. J., Hoyt, S. C., and Tanigoshi, L. K. 1976. An analytic model for description of temperature dependent rate phenomena in arthropods. Environ. Entomol. 5:1133-1140.

6. Newton, M. R., Wright, A. S., Kinkel, L. L., and Leonard, K. J. 1999. Competition alters temporal dynamics of sporulation in the wheat stem rust fungus. J. Phytopathol. 147:527-534.

7. Roelfs, A. P. 1985. Wheat and rye stem rust. Pages 3-59 in: The Cereal Rusts, vol. 2. Diseases, Distribution, Epidemiology, and Control. A. P. Roelfs and W. R. Bushnell, eds. Academic Press, New York.

8. Roelfs, A. P., Singh, R. P., and Saari, E. E. 1992. Rust diseases of wheat: Concepts and methods of disease management. International Maize and Wheat Improvement Center, Mexico, D.F.

9. Schroedter, H. 1965. Methodisches zur bearbeitung phytometeoro-pathologischer untersuchungen dargestellt am beispiel der temperaturrelation. Phytopathol. Z. 53:154-166.

10. Shaner, G. 1980. Probits for analyzing latent period data in studies of slow rusting resistance. Phytopathology 70:1179-1182.

11. Shaner, G., and Hess, F. D. 1978. Equations for integrating components of slow leaf-rusting resistance in wheat. Phytopathology 68:1464-1469.

12. Teng, P. S., Blackie, M. J., and Close, R. C. 1980. Simulation of the barley leaf rust epidemic: Structure and validation of BARSIM-I. Agric. Syst. 5:85-103.

13. Webb, D. H., and Nutter, F. W., Jr. 1997. Effects of leaf wetness duration and temperature on infection efficiency, latent period, and rate of pustule appearance of rust in alfalfa. Phytopathology 87:946-950.

14. Welty, R. E., and Barker, R. E. 1992. Latent-period responses of stem rust in tall fescue incubated at four temperatures. Crop Sci. 32:589-592.

15. Wilcoxson, R. D. 1981. Genetics of slow rusting in cereals. Phytopathology 71:989-993.

16. Wilson, J., and Shaner, G. 1989. Individual and cumulative effects of long latent period and low infection type reactions to Puccinia recondita in Triticale. Phytopathology 79:101-108. 